\title{
Recurrence of Space-Time Events
}

\author{
Nasr Ahmed 1,2 \\ ${ }^{1}$ Astronomy Department, National Research Institute of Astronomy and Geophysics, Helwan, Cairo, Egypt \\ ${ }^{2}$ Mathematics Department, Faculty of Science, Taibah University, Madina, Saudi Arabia \\ Email: nasr.ahmed@nriag.sci.eg, abualansar@gmail.com
}

Received 9 August 2015; accepted 14 October 2015; published 19 October 2015

Copyright () 2015 by author and Scientific Research Publishing Inc.

This work is licensed under the Creative Commons Attribution International License (CC BY).

http://creativecommons.org/licenses/by/4.0/

(c) (i) Open Access

\begin{abstract}
A causal-directed graphical space-time model has been suggested in which the recurrence phenomena that happen in history and science can be naturally explained. In this Ramsey theorem inspired model, the regular and repeated patterns are interpreted as identical or semi-identical space-time causal chains. The "same colored paths and subgraphs" in the classical Ramsey theorem are interpreted as identical or semi-identical causal chains. In the framework of the model, Poincare recurrence and the cosmological recurrence arise naturally. We use Ramsey theorem to prove that there's always a possibility of predictability no matter how chaotic the system is.
\end{abstract}

\section{Keywords}

\section{Space-Time Models, Causal Directed-Graphs, Chaotic Systems, Recurrence Phenomena}

\section{Introduction}

\subsection{Historical Recurrence}

History is a record of past events. In the literature, historical recurrence is an hypothetical concept which refers to the repetition of similar events in history. This concept, sometimes referred to as history repeats itself, has attracted the attention of many thinkers and authors through the history with the absence of any rigorous scientific base to explain it [1]-[5]. It might be best expressed in Mark Twain's words that no occurrence is sole and solitary, but is merely a repetition of a thing which has happened before, and perhaps often [6]. For some historic examples see [1] where Trompf traced historically recurring patterns of political thought and behavior in the west since antiquity. The recurrence idea also exists in science and has been discussed in different contexts. In dynamical systems, Poincare's recurrence theorem [7] states that, in general, all systems will return not once but infinitely many times to a configuration that is very close to its initial one. The time elapsed until the recurrence is the Poincare recurrence time and depends on the exact initial state and required degree of closeness [8]. A quantum recurrence theorem which is a quantum analog of Paincare's recurrence theorem was proved in 
1975 [9]. Birkhoff's recurrence theorem (1972) states: if $X$ is a compact metric space and $T$ is a continuous map of $X$ into itself, there exists some point $x_{o} \in X$ and some sequence $n_{k} \rightarrow \infty$ with $T^{n_{k}} x_{o} \rightarrow x_{o}$.

In geophysics, the study of the recurrence of the earthquake events is very important. It has been shown in [10] that the recurrence intervals between earthquake events strongly depend on history. Distribution of the recurrence times and the mean residual time until the next earthquake strongly depends on the previous recurrence time. In cosmology, several recurrence models have been introduced in which the evolution of the universe is cyclic (Ekpyrotic and cyclic cosmology). The name Ekpyrotic represents the contractive phase of eternallyrecurring destruction and re-creation [11] [12]. In [13], a cosmological model has been proposed in which the universe undergoes an endless sequence of cosmic epochs each beginning with a bang and ending in a crunch.

\subsection{Ramsey Theory}

Ramsey theory is the study of unavoidable regularity in large structures. It is a foundational result in combinatorics named after Frank Ramsey who did seminal work in this area before his death in 1930 [14]. The theory was developed extensively by Erdos [15].

The original theorem proved by Ramsey could be written as: In any colouring of the edges of a sufficiently large complete graph, one will find monochromatic complete subgraphs. So, no matter how chaotic we try to arrange certain objects, we will find ourselves creating a very highly organized and structured object within it. The quote "complete disorder is impossible" describing Ramsey theory is attributed to Theodore Motzkin [16].

The Infinite version of Ramsey's theorem states that: If $X$ is a countably infinite set and for any $n \in N$ the subsets of size $n, X^{n}$, are colored in infinitely many colors, then there is an infinite set $M \subseteq X$ where all subsets of $M$ of size $n$ are colored in the same color.

For the graphs $G_{1} ; G_{2} ; \cdots ; G_{t}$, the graph Ramsey number $r\left(G_{1} ; G_{2} ; \cdots ; G_{t}\right)$ is the smallest integer $R$ with the property that any complete graph of at least $R$ vertices whose edges are partitioned into $t$ color classes contains a monochromatic subgraph isomorphic to $G_{i}$ in the $i$-th color for some $i, 1 \leq i \leq t$. These are classical Ramsey numbers or simply Ramsey numbers when all graphs are complete graphs which correspond to the original definition, later extended to any graph [17].

Ramsey theorem has been a useful tool in many areas of mathematics. There have also been some attempts to use graph theory in social sciences (see [18] as an example). Some attempts to use Ramsey theory in the study of history has been mentioned by Gasarch in [19]. The classical Ramsey Theorem has since been generalized in many ways; most of these generalizations are concerned with finding monochromatic substructures in various colored structures [20]-[23]. Monochromatic paths and cycles have been investigated by many authors, for example see [24]-[26].

It's useful to quickly recall some basic definitions: The complete graph $K_{n}$ of order $n$ is a simple graph with $n$ vertices in which an edge connects every pair of vertices. A clique in a graph is a set of pairwise adjacent vertices. Since any subgraph induced by a clique is a complete subgraph, the two terms and their notations are usually used interchangeably. A path from vertex $V_{0}$ to vertex $V_{k}$ is a sequence $V_{0} ; E_{0} ; V_{1} ; E_{1} ; V_{2}$; $E_{2} ; \ldots ; V_{k-1} ; E_{k-1} ; V_{k}$ of vertices $V_{k}$ and edges $E_{k-1}$. A cycle in a graph is a path from some vertex $V_{0}$ back to $V_{0}$ (a closed path) where no edge appears more than once in the path sequence. A directed graph is one in which the direction of any given edge is defined. Conversely, in an undirected graph we can move in both directions between vertices. Two graphs are isomorphic when the vertices of one can be relabeled to match the vertices of the other in a way that preserves adjacency.

\section{Space-Time Causal Chains}

Starting with the fact that every space-time event can be represented as a point in the four dimensional spacetime manifold. We consider the space-time "macroscopic" events as a random distribution of countably infinite space-time points. In this system causality can not be violated and hence the arrow of time must be respected. The points are connected to each other by edges which represent the causal relations and we are allowed to move from one point to another only in the future direction, i.e. edges are future directed time-like vectors. So we actually have a partially connected network or a graph with subgraphs and paths. Since some events are related to each others and some are not, it can not be a complete graph or fully connected network. In this spacetime event graph, no self-loops are allowed. Loops are edges connected at both ends to the same event which implies the existence of closed time-like loops in the space-time manifold allowing traveling to the past [27]. 
The kind of graphs which guarantee the fulfillment of the arrow of time condition with no loops are the Directed acyclic graph (DAG). That kind of graphs are sometimes referred to as causal graphs or path diagrams. Causal-directed graphical models were developed in the philosophy of science and statistical literature, they are also used in epidemiology, genetics and related disciplines (see [28] and references therein). Denoting edges by $E$ and vertices by $V$ we can set the following definition:

Definition: A space-time directed acyclic graph $G$ is a pair $(V, E)$ where $V$ is a set of events and $E$ is a set of causal relations $E \subseteq\left\{\left(V_{1}, V_{2}\right) \mid V_{1}, V_{2} \in V\right\}$.

Where an ordered pair of two events $\left(V_{1}, V_{2}\right)$ defines "the space-time event arc" directed from $V_{1}$ to $V_{2}$. Every directed path defines a space-time causal chain. A space-time causal chain is defined as an ordered sequence of events in which any one event in the chain causes the next.

Ramsey numbers for directed graphs were introduced by Erdos and Moser [29] [30]. The directed analogue of the two possible arc colours is the two directions of the arcs and the analogue of "monochromatic" is that all arcarrows point the same way i.e. acyclic.

Applying Ramsey theorem to this countably infinite chaotic distribution of space-time events implies the existence of regular and repeated patterns in the form of identical and semi-identical paths and subgraphs, i.e. identical space-time causal chains (Figure 1). Having a countably infinite number of edges connecting randomly distributed countably infinite number of points makes the existence of identical paths and cliques guaranteed. Identical directed paths correspond to identical space-time causal chains. We could state the following conjecture:

Conjecture: For a countably infinite number of space-time events, there is a countably infinite number of identical or semi-identical causal chains.

The identical and semi-identical causal chains are explained by the striking similarities among events and sequences of events at different space-time locations. This is just what is called historic recurrence which is clearly observable in the study of human history. According to that, the repetition of similar events in history is unavoidable. Similar analysis is applicable for dynamical systems as we are going to see in the next sections

\section{Predictability and Evolving Systems}

How possible is it to predict a future causal chain that is identical to a past causal chain before it happens? The

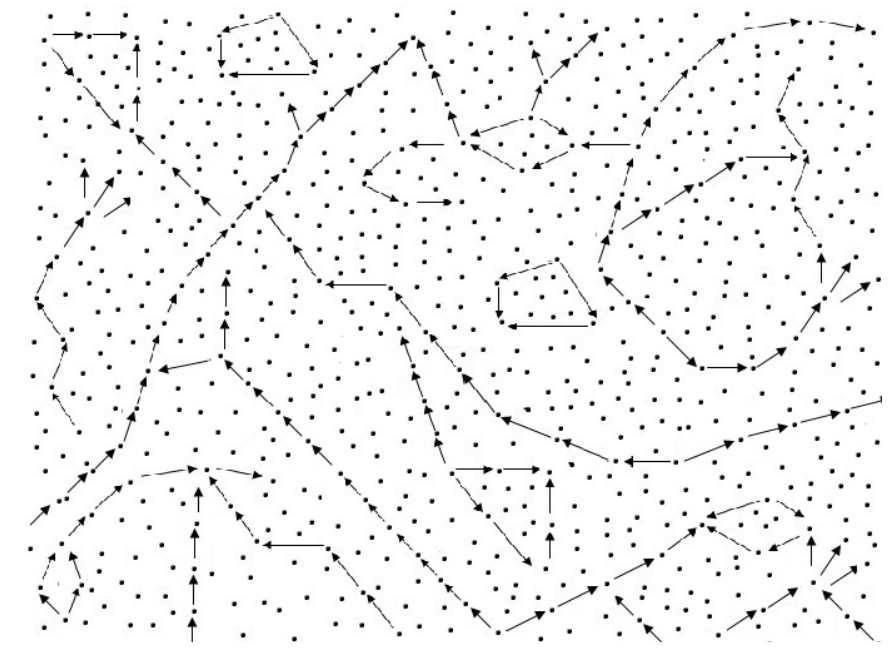

Figure 1. Macroscopic causal chains with the vertices represent the events and the edges are future directed time-like vectors. For example, three arrows directed toward a point means three reasons caused that event and so on. For a countably infinite number of events, the existence of identical causal chains is guaranteed. Identical directed paths and subgraphs are interpreted as identical causal chains at different space-time locations. Historic recurrence hypothesis and some recurrence theorems such as Poincare and cosmological recurrence have a natural interpretation here. 
predictability of macroscopic space-time events is very different from the predictability of point particles in dynamical systems where we easily predict the particle's future motion. Suppose we have two causal chains $A_{1} \rightarrow A_{2} \rightarrow A_{3} \rightarrow A_{4}$ and $B_{1} \rightarrow B_{2} \rightarrow B_{3} \rightarrow B_{4}$. The predictability strength of a certain future event depends on the degree of simialrity between the previous events in the two chains. Here, the more similar the two events $A_{3}$ and $B_{3}$ are, the more similar the two events $A_{4}$ and $B_{4}$ will be. Exceptions happen due to the nature of human beings as living creatures with a will. However, there is always a possibility to predict the future events and this is what we are going to prove in this section by making use of Ramsey theorem.

For a given space-time location, different events happen at different times. So there is a flow of events going through each space-time location. We consider the general case of a moving disorder; chaos. We need to investigate the degree of predictability for a system of an infinite number of points moving chaotically. We start by setting the following definition relating the degree of predictability of any dynamical system to its degree of disorder:

Definition: A dynamical system is said to be completely unpredictable if and only if it is in a complete disorder.

Theorem complete unpredictability is impossible.

Proof:

Proposition: The system is completely unpredictable.

For this system, at any fixed time $t$, we get a static disordered distribution of points for which Ramsey theorem is valid, so this static snapshot of the system is not in a complete disorder. This is valid for any snapshot taken at any time $t_{i}$ during the evolution of the system.

Denoting the system's degree of disorder by $S$ and “the possibility of predictability" by $P(S)$, we have $\forall t_{i} \exists P(S)$. But from the definition, this contradicts with our proposition about the complete unpredictability of the system. So the proposition is false and the complete unpredictability of this chaotic system is impossible. The proof is valid for space-time future events and for dynamical systems, too.

\section{Other Recurrence Theorems Revisited}

Both Poincare's recurrence and the cosmological recurrence can find a natural interpretation in the framework of the causal-directed graphical space-time model. In Poincare's recurrence theorem, systems will return not once but infinitely many times to a configuration very close to the initial one. This simply will happen as the system passes through a causal chain that is identical or semi-identical to its initial one. Since there is a countably infinite number of identical or semi-identical causal chains, the system will return infinitely many times to a configuration very close to its initial one.

The same analysis applies for the cosmological recurrence. The recurrence will happen when the universe passes through a causal chain that is identical to its initial one.

\section{Conclusion}

A directed acyclic space-time graph model has been suggested in which a natural explanation of the long standing hypothesis of historic recurrence could be found. Applying Ramsey theorem on a countably infinite or suffeciently large number of space-time events emphasizes the existence of regular patterns in the causal structure of space-time events. The suggested analysis shows that historic recurrence might not be just a hypothetical concept but a necessity associated with any sufficiently large number of space-time events. Other recurrence theorems can also be naturally explained such as Poincare recurrence and cosmological recurrece. There is always a possibility of predictability depending on the system's degree of disorder.

\section{References}

[1] Trompf, G.W. (1979) The Idea of Historical Recurrence in Western Thought, from Antiquity to the Reformation. University of California Press, Berkeley.

[2] Toynbee, A.J. (1948) Does History Repeat Itself? Civilization on Trial. Oxford University Press, New York.

[3] Kennedy, P. (1987) The Rise and Fall of the Great Powers: Economic Change and Military Conflict from 1500 to 2000. Random House, New York.

[4] Graham, G. (1997) The Shape of the Past: A Philosophical Approach to History. Oxford University Press, Oxford. http://dx.doi.org/10.1093/acprof:0so/9780192892553.001.0001 
[5] Sorokin, P.A. (1957) Social and Cultural Dynamics: A Study of Change in Major Systems of Art, Truth, Ethics, Law, and Social Relationships. Porter Sargent Publishing, Boston.

[6] Twain, M. (1903) The Jumping Frog. Harper and Brothers, New York.

[7] Barreira, L. (2006) Poincaré Recurrence: Old and New. Proceedings of the 14th International Congress on Mathematical Physics, Lisbon, 28 July-2 August 2003, 415-422. http://dx.doi.org/10.1142/9789812704016_0039

[8] Brush, S.G. (1996) A History of Modern Planetary Physics: Nebulous Earth V1. Cambridge University Press, Cambridge.

[9] Bocchieri, P. and Loinger, A. (1957) Quantum Recurrence Theorem. Physical Review, 107, 337-338. http://dx.doi.org/10.1103/PhysRev.107.337

[10] Livina, V., Tuzov, S., Havlin, S. and Bunde, A. (2005) Recurrence Intervals between Earthquakes Strongly Depend on History. Physica A: Statistical Mechanics and its Applications, 348, 591- 595. http://dx.doi.org/10.1016/j.physa.2004.08.032

[11] Lehners, J.-L. (2008) Ekpyrotic and Cyclic Cosmology. Physics Reports, 465, 223-263. http://dx.doi.org/10.1016/j.physrep.2008.06.001

[12] Penrose, R. (2011) Cycles of Time: An Extraordinary New View of the Universe. Alfred Knopf, New York.

[13] Steinhardt, P.J. and Turok, N. (2001) A Cyclic Model of the Universe. Science, 296, 1436-1439. http://dx.doi.org/10.1126/science.1070462

[14] Ramsey, F.P. (1930) On a Problem of Formal Logic. Proceedings of the London Mathematical Society, s2-30, $264-286$. http://dx.doi.org/10.1112/plms/s2-30.1.264

[15] Halasz, et al., Eds. (2002) Paul Erds and His Mathematics. Springer, Berlin.

[16] Prömel, H.J. (2005) Complete Disorder Is Impossible: The Mathematical Work of Walter Deuber. Combinatorics, Probability and Computing, 14, 3-16.

[17] Burr, S.A. (1974) Generalized Ramsey Theory for Graphs-A Survey. In: Bari, R. and Harary, F., Eds., Graphs and Combinatorics, Springer, Berlin, 52-75.

[18] Cartwright, D. and Harary, F. (1977) A Graph Theoretic Approach to the Investigation of System-Environment Relationships. Journal of Mathematical Sociology, 5, 87-111. http://dx.doi.org/10.1080/0022250X.1977.9989866

[19] http://www.cs.umd.edu/ gasarch/BLOGPAPERS/ramseykings.pdf

[20] Graham, R.L., Rothschild, B.L. and Spencer, J.H. (1990) Ramsey Theory: Wiley-Interscience Series in Discrete Mathematics and Optimization. 2nd Edition, John Wiley and Sons, Inc., New York.

[21] Kittipassorn, T. and Narayanan, B.P. (2014) A Canonical Ramsey Theorem for Exactly m-Coloured Complete Subgraphs. Combinatorics, Probability and Computing, 23, 102-115. http://dx.doi.org/10.1017/S0963548313000503

[22] Folkman, J. (1970) Graphs with Monochromatic Complete Subgraphs in Every Edge Coloring. SIAM Journal on Applied Mathematics, 18, 19-24. http://dx.doi.org/10.1137/0118004

[23] Bollobás, B. and Gyárfás, A. (2008) Highly Connected Monochromatic Subgraphs. Discrete Mathematics, 308, 1722-1725. http://dx.doi.org/10.1016/j.disc.2006.01.030

[24] Scott, A. and White, M. (2011) Monochromatic Cycles and the Monochromatic Circumference in 2-Coloured Graphs. http://arxiv.org/abs/1107.5177

[25] Benevides, F., Luczak, T., Skokan, J., Scott, A. and White, M. (2012) Monochromatic Cycles in 2-Coloured Graphs. Combinatorics, Probability and Computing, 21, 57-87. http://dx.doi.org/10.1017/S0963548312000090

[26] Gyárfás, A. (1983) Vertex Coverings by Monochromatic Paths and Cycles. Journal of Graph Theory, 7, 131-135. http://dx.doi.org/10.1002/jgt.3190070116

[27] Hawking, S.W. and Ellis, G.F.R. (1973) The Large-Scale Structure of Space-Time. Cambridge University Press, Cambridge. http://dx.doi.org/10.1017/CBO9780511524646

[28] Gopnik, A. and Schulz, L. (2007) Causal Learning: Psychology, Philosophy and Computation. Oxford University Press, New York. http://dx.doi.org/10.1093/acprof:oso/9780195176803.001.0001

[29] Erds, P. and Moser, L. (1964) On the Representation of Directed Graphs as Unions of Orderings. Publications of the Mathematical Institute of the Hungarian Academy of Sciences, 9, 125-132.

[30] Bermond, J.C. (1974) Some Ramsey Numbers for Directed Graphs. Discrete Mathematics, 9, 313-321. http://dx.doi.org/10.1016/0012-365X(74)90077-6 